\title{
CETOACIDOSE DIABÉTICA E ESTADO HIPERGLICÊMICO HIPEROSMOLAR
}

\author{
DIABETIC KETOACIDOSIS AND HYPEROSMOLAR HYPERGLYCEMIC STATE
}

Maria C. Foss-Freitas ${ }^{1} \&$ Milton C. Foss ${ }^{2}$

\begin{abstract}
${ }^{1}$ Pós-Graduanda. ${ }^{2}$ Docente. Divisão de Endocrinologia e Metabologia. Departamento de Clínica Médica. Faculdade de Medicina de Ribeirão Preto - USP

CoRRESPONDÊNCIA: Milton Cesar Foss. Hospital das Clínicas-Faculdade de Medicina de Ribeirão Preto-USP. Av. Bandeirantes,3900 CEP 14049-900 Ribeirão Preto-SP-Brasil - FONE (16) 602-2467 - FAX (16) 633-6695 - E-mail mcfoss@fmrp.usp.br.
\end{abstract}

FOSS-FREITAS MC \& FOSS MC. Cetoacidose diabética e estado hiperglicêmico hiperosmolar. Medicina, Ribeirão Preto, 36: 389-393, abr./dez. 2003.

RESUMO - A cetoacidose diabética é uma complicação aguda, típica dos pacientes com DM tipo I ou insulinodependentes, um conjunto de distúrbios metabólicos, que se desenvolvem em uma situação de deficiência insulínica grave, comumente associada a condições estressantes, que levam a aumento dos hormônios contra-reguladores. O estado hiperglicêmico hiperosmolar é uma complicação aguda, característica do diabético tipo II ou não insulinodependente, quando predominam os efeitos da hiperosmolaridade e desidratação, principalmente envolvendo o sistema nervoso central. Fatores precipitantes, quadro clínico, fisiopatologia básica e tratamento dessas emergências médicas são analisados nesta revisão.

UNITERMOS - Diabetes Mellitus Tipo 1. Diabetes Mellitus Tipo 2. Cetoacidose Diabética. Estado Hiperglicêmico Hiperosmolar. Diabetes Mellitus Insulino-Dependente. Diabetes Mellitus Não Insulino-Dependente.

As complicações agudas, hiperglicêmicas, do paciente diabético ainda representam um importante problema de saúde pública nas unidades de emergência, tanto em nosso meio como em âmbito mundial. Particularmente, a cetoacidose diabética tem sido registrada com freqüência significativa e crescente, na última década, em estudos de incidência nos Estados Unidos e em alguns países europeus, o que também tem sido observado em nossas unidades de emergência. Felizmente, as taxas de mortalidade relacionadas a essas agudas complicações diabéticas têm diminuído, incluindo os pacientes em estado hiperglicêmico hiperosmolar, e, quando ocorrem mortes, estão, geralmente, associadas a graves infecções generalizadas.

A cetoacidose é uma complicação aguda, típica do paciente diabético do tipo 1, e esse conjunto de distúrbios metabólicos se desenvolve em uma situação de deficiência insulínica grave ou absoluta, comumente associada a condições estressantes, que levam ao aumento dos hormônios contra-reguladores. $\mathrm{O}$ aumento da atividade cetogênica é um componente fisiopatológico marcante em tal situação de emergência clínica. $\mathrm{O}$ estado hiperglicêmico hiperosmolar é uma complicação aguda, característica do diabético tipo 2, com deficiência insulínica relativa e que se caracteriza pela hiperglicemia, hiperosmolaridade e desidratação, principalmente envolvendo o sistema nervoso central.

Os fatores precipitantes da cetoacidose ou do estado hiperglicêmico hiperosmolar são, em sua maioria, de natureza infecciosa aguda (respiratória, urinária, genital, cutânea, etc.), freqüentemente associados 
ou não, ao tratamento insulínico interrompido ou inadequado às condições do paciente. Situações agudas estressantes, tanto de causa emocional isolada como acompanhando quadros orgânicos graves de acidentes vasculares (cerebrais ou coronarianos), pancreatites agudas, etc. Também têm sido associadas ao desencadeamento destas complicações diabéticas. Não é raro o desenvolvimento de situações de descompensação metabólica aguda, principalmente a cetoacidose, em pacientes jovens, no início do quadro diabético, sem diagnóstico prévio.

O entendimento do quadro clínico da cetoacidose diabética (CAD) fundamenta-se no conhecimento da fisiopatologia básica dessa situação clínica, que tem, como elemento fundamental, uma deficiência insulínica, absoluta, marcante, geralmente associada a aumento dos hormônios antagonistas (glucagon, GH, glicocorticóides, catecolaminas). A hiperglicemia, resultante tanto da diminuição da utilização periférica de glicose como do aumento de sua produção endógena (glicogenólise e neoglicogênese), é um componente fisiopatológico característico da situação, justificando vários dos sintomas e sinais típicos da CAD, como polidipsia, poliúria e graus variados de desidratação, que podem chegar à hipovolemia acentuada $\mathrm{e}$ choque circulatório. Os distúrbios eletrolíticos também se desenvolvem por perda renal excessiva, levando a deficiências marcantes de $\mathrm{NA}+, \mathrm{K}+, \mathrm{Cl}-, \mathrm{PO} 4(, \mathrm{Ca}++$, $\mathrm{Mg}++$, no organismo, que podem se acentuar pela presença freqüente de náuseas e vômitos nos pacientes. É comum a observação de dor abdominal, provavelmente devida aos distúrbios hidroeletrolíticos e metabólicos da cetoacidose, que, geralmente, melhora, com o tratamento do paciente. $\mathrm{O}$ aumento da cetogênese hepática, aliada à lipólise acentuada e maior mobilização de ácidos graxos, livres ao fígado, é a base fisiopatológica da hipercetonemia e das alterações do equilíbrio acidobásico desses pacientes diabéticos. Os corpos cetônicos, acetoacetato(AcAc) e 3- $\beta$-hidróxidobutirato( $3 \mathrm{HB})$, são produzidos durante a cetogênese hepática e usados como fontes alternativas de energia. Estão presentes na corrente sanguínea, na proporção de 13HB:1AcAc, e seus níveis podem aumentar durante o jejum e o exercício físico. Na deficiência insulínica absoluta, seus níveis se elevam, podendo apresentar um aumento de até 10:1. Como o aumento do 3HB é mais significativo, torna-se importante ressaltar que os métodos que utilizam o nitroprussiato de sódio para a quantificação dos corpos cetônicos detectam somente o AcAc e que, durante o tratamento da $\mathrm{CAD}$, os níveis de $3 \mathrm{HB}$ têm queda mais tardia em relação ao AcAc.

O desenvolvimento da cetoacidose é progressivo, evoluindo de cetose inicial com acidose compensada até graus avançados de hipercetonemia e acidose metabólica, com manifestações típicas de hálito cetônico e alterações respiratórias compensatórias (respiração de Kussmaul - respiração ampla e acelerada). O conjunto das alterações hidroeletrolíticas e metabólicas pode ter graus variados de repercussão sobre a função do sistema nervoso central, podendo determinar desde graus leves de sonolência, torpor e confusão mental até o estabelecimento de estado de coma profundo. Assim o diagnóstico da cetoacidose, em paciente diabético, fundamenta-se no entendimento de toda a apresentada situação clínica, com sintomas e sinais característicos, e exige do médico assistente uma atitude rápida e eficiente na sua observação clínica (anamnese e exame físico) : desenvolvimento do quadro clínico e fatores precipitantes, caracterização do estado hidroeletrolítico do paciente e envolvimento de outros sistemas e órgãos no quadro de cetose e acidose metabólica.(Figura 1)

O estado hiperglicêmico hiperosmolar (EHH), uma complicação aguda, típica do diabético Tipo 2, caracteriza-se por uma descompensação grave do estado diabético com uma taxa de mortalidade ainda muito significativa. O quadro clínico dessa condição manifesta sinais e sintomas de hiperglicemia e hiperosmolaridade acentuadas, desidratação grave, com envolvimento, em grau variável, do sistema nervoso central. O paciente típico de tal complicação é, geralmente, idoso e adentra as unidades de emergência por acentuação das alterações de consciência, crises convulsivas e sintomas sugestivos de acidentes vasculares cerebrais. Os sinais de desidratação grave, levando a alterações sensoriais graves e choque circulatório, são sempre muito evidentes ao exame físico do paciente. Os exames laboratoriais iniciais são os mesmos indicados para o paciente diabético que chega em CAD, porém, o quadro é não cetótico (cetonúria negativa ou fracamente positiva (+)), devido à presença de quantidades suficientes de insulina para bloquear a cetogênese hepática.

Os exames laboratoriais, indicados antes do início do tratamento do paciente são:

Glicemia - Sempre aumentada, podendo apresentar variações amplas, sendo que, nos casos de EHH, chega a valores acima de $1000 \mathrm{mg} / \mathrm{dl}$. 


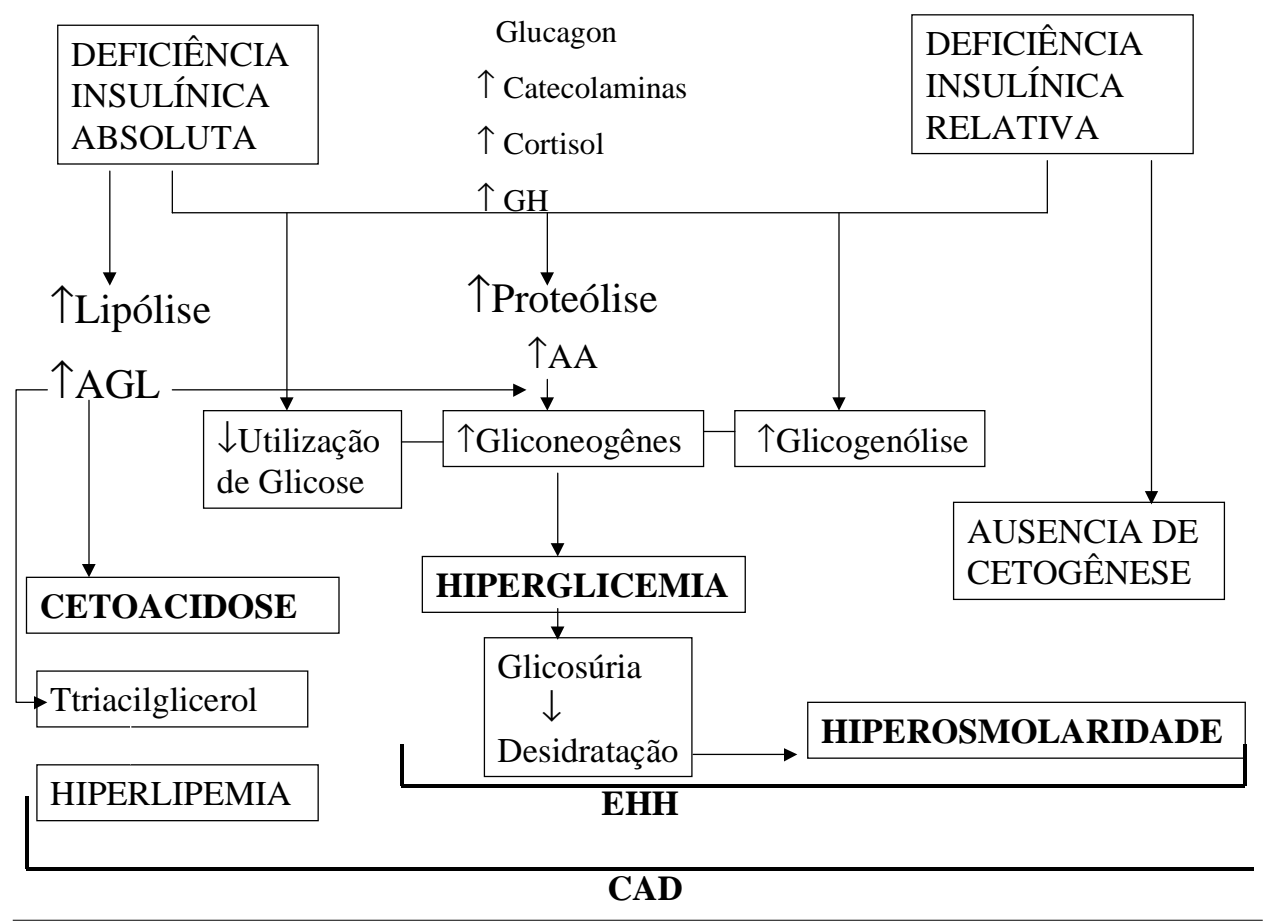

Figura 1: Representação esquemática da fisiopatologia da cetoacidose diabética e do estado hiperglicêmico hiperosmolar

Glicosúria/Cetonúria - Na CAD, os testes são positivos, geralmente, em níveis máximos dos métodos semiquantitativos utilizados: glicofitas ou métodos realizados em laboratórios de análise. $\mathrm{Na} \mathrm{EHH}$, a cetonúria é negativa ou fracamente positiva $(+)$.

Eletrólitos - $\mathrm{Na}+\mathrm{K}+$ : expoliação eletrolítica ( $\mathrm{Na}+, \mathrm{K}+, \mathrm{Cl}-, \mathrm{PO} 4(, \mathrm{Ca}++, \mathrm{Mg}++$, etc.) está bem caracterizada na CAD e EHH, gerando deficiências, geralmente, muito acentuadas. A perda hídrica associada determina níveis variáveis para os eletrólitos sanguíneos (altos, normais ou baixos). Níveis diminuídos dos eletrólitos nessas condições indicam carências graves.

Gasometria arterial - Na avaliação do equilíbrio acidobásico dos pacientes, três parâmetros são fundamentais :

1. pH sanguíneo : diminuído (ácido,) quando as reservas tamponantes do sangue são consumidas (nor$\mathrm{mal}=7,4(0,05)$;

2. bicarbonato (BS) : que diminui à medida que a produção de ácidos orgânicos vai aumentando (nor$\mathrm{mal}=24(2 \mathrm{mEq} / \mathrm{l})$;

3. base excess (BE): diminuída com o consumo de bases (normal $=+2,5 \mathrm{a}-2,5 \mathrm{mEq} / \mathrm{l}$ ).

Uréia/Creatinina: podem se elevar na cetoacidose pelo catabolismo protéico e desidratação, mas valores significativamente aumentados podem indicar insuficiência renal prévia ou surgida no curso da CAD ou EHH (desidratação-choque circulatório).

Hematológico: leucocitose com neutrofilia e hematócrito aumentado são comumente observados na CAD ou EHH, entretanto, é um exame útil na caracterização de fatores infecciosos, precipitantes do quadro metabólico.

Considerando-se os fatores precipitantes de urgências hiperglicêmicas, também podem ser indicados: urina rotina, culturas (urina, sangue, etc.), exames radiológicos, etc.

O tratamento da CAD, ou EHH, fundamenta-se na correção dos distúrbios endocrinometabólicos, hidroeletrolíticos e acidobásicos do paciente, com as linhas gerais de conduta propostas a seguir:

\section{Paciente adulto}

a) Líquidos - administração EV na seguinte seqüência: 011 de soro fisiológico em $01 \mathrm{~h}$, 011 de soro fisiológico em $01 \mathrm{~h}$, 011 de soro fisiológico em $02 \mathrm{~h}$, 011 de soro fisiológico em $04 \mathrm{~h}$, $011 \mathrm{de}$ soro fisiológico em $04 \mathrm{~h}$, $011 \mathrm{de}$ soro fisiológico em $08 \mathrm{~h}$.

\section{Atenção}

- Se o paciente for idoso e/ou apresentar doença cardiovascular, a reposição hídrica deve ser feita com controle de P.V.C.

- Se o $\mathrm{Na}+$ plasmático for maior que $155 \mathrm{mEq} / \mathrm{l}$, trocar o S.F. $(0,9 \%)$ por S.F. hipotônico $(0,45 \%)$. - Quando a glicemia cair abaixo de $250 \mathrm{mg} / \mathrm{dl}$, trocar o S.F. $(0,9 \%)$ para solução glicosilada $5 \%$ ou glicofisiológica 5\%, se o $\mathrm{Na}+$ plasmático ainda estiver baixo.

b) Insulina - administrar inicialmente 10-20U. de insulina simples I.M. (dose ataque) e, a seguir, $5 \mathrm{U}$. 
I.M. por hora, até a glicemia cair abaixo de 250 $\mathrm{mg} / \mathrm{dl}$ (glicosúria + ou negativa)na CAD e $300 \mathrm{mg} /$ dl no EHH, quando se deve passar a 5U. I.M., a cada $02 \mathrm{~h}$ ou, posteriormente, a cada $04 \mathrm{~h}$, até o paciente voltar a se alimentar com esquema fixo de insulina às refeições.

c) Potássio - administrar, inicialmente, $13 \mathrm{mEq} / \mathrm{h}$, desde a $1^{\mathrm{a}}$ injeção de insulina, usando conexão $\mathrm{Y}$ com equipo do soro fisiológico (diluir 04 ampolas de $10 \mathrm{ml}$ de $\mathrm{KCl} 20 \%$ em $500 \mathrm{ml} \mathrm{S.F.}-1 \mathrm{ml}(20$ gts $) / \mathrm{min} .=60 \mathrm{ml} / \mathrm{h}=13 \mathrm{mEq} / \mathrm{h}$ ). Com as dosagens laboratoriais, obtidas (se possível) no prazo de até $1 \mathrm{~h}$ após a colheita, proceder da seguinte forma:

$$
\begin{array}{ll}
4<\quad & \mathrm{K}+(4 \mathrm{mEq} / \mathrm{l} \text {, passar para } 26 \mathrm{mEq} / \mathrm{h} \\
5 & \mathrm{~K}+(5 \mathrm{mEq} / \mathrm{l} \text {, manter para } 13 \mathrm{mEq} / \mathrm{h} \\
\mathrm{K}+(6 \mathrm{mEq} / \mathrm{l} \text {, passar para } 6,5 \mathrm{mEq} / \mathrm{h} \\
\mathrm{K}+>6 \mathrm{mEq} / \mathrm{l} \text {, suspender a infusão }
\end{array}
$$

d) Bicarbonato de sódio - administrar NaHCO3. E.V., se $\mathrm{pH}$ arterial $<7,0$ após o início da reposição hídrica e insulinoterapia, na dose de $100 \mathrm{mEq}$ em $01 \mathrm{~h}$.

Medir pH arterial 30 min após a administração de bicarbonato. Se o pH continuar abaixo de 7,0, repetir a infusão.

(Solução de NaHCO3, 10\% tem 1,2 mEq/ml)

\section{Crianças}

a) Líquidos - O grau da desidratação deverá ser estimado clinicamente $-2,5$ a $12 \%$ em crianças até a idade pré-escolar e 03 a $09 \%$ em crianças maiores, e o volume correspondente deve ser administrado em 06 h. Em caos de hipovolemia ou má perfusão periférica deve-se infundir $20 \mathrm{ml} / \mathrm{kg}$ na primeira hora e o restante em 05 horas.

A composição da solução deverá ser a seguinte :

- lactentes : solução de $\mathrm{NaCl} 0,9 \%$ : água destilada $(1: 1)$.

- crianças maiores : solução de $\mathrm{NaCl} 0,9 \%$ : água destilada $(2: 1)$.

Atenção: Se $\mathrm{Na}$ + plasmático for maior que 155 $\mathrm{mEq} / \mathrm{l}$ ou a osmolaridade plasmática calculada

$$
\left\{2(\mathrm{Na}++\mathrm{K}+)+\frac{\text { Glicose }}{18}+\frac{\mathrm{BUN}}{2,8}\right\}
$$

maior que $350 \mathrm{mOsm} / \mathrm{l}$, trocar o S.F. $(0,9 \%)$ por solução de $\mathrm{NaCl}$ hipotônica $(0,45 \%)$.
Quando a glicemia cair abaixo de $250 \mathrm{mg} / \mathrm{dl}$, equivalente a aproximadamente + de glicosúria, substituir o soro fisiológico por soro glicofisiológico $05 \%$, se a criança ainda estiver desidratada. Caso contrário, passar para a solução de manutenção $(1 / 4 \mathrm{NaCl}$ $0,9 \%: 3 / 4$ SG $5 \%$ ).

b) Insulina - Administrar, inicialmente, $0,25 \mathrm{U} / \mathrm{Kg}$ peso de insulina simples I.M. (dose de ataque). A seguir $0,1 \mathrm{U} / \mathrm{Kg}$ de peso I.M. por hora até a glicemia cair a menos de $250 \mathrm{mg} / \mathrm{dl}$, quando se deve-introduzir soro glicosado e passar a 0,05 a $0,1 \mathrm{U} / \mathrm{Kg}$ I.M. cada $02 \mathrm{~h}$ ou, posteriormente, a cada $04 \mathrm{~h}$. Quando o paciente voltar a se alimentar, iniciar esquema fixo de múltiplas doses de insulina simples, antes das refeições $(0,1$ e $0,3 \mathrm{U} / \mathrm{Kg}$ peso). Finalmente, inicia-se o tratamento com insulina $\mathrm{NPH}$, pela manhã, 0,5 a $1 \mathrm{U} / \mathrm{Kg}$ ou $70 \%$ da dose necessária nas últimas $24 \mathrm{~h}$.

Na cetoacidose, quando a glicemia atingir 250 $\mathrm{mg} / \mathrm{dl}$, deve-se manter insulinoterapia e soro glicosado até a cetonúria negativar. Nesse período, a faixa de glicemia desejável deverá estar em torno de 200 mg/ dl.

c) Potássio - Administrar $\mathrm{K}+$ desde a $1^{\mathrm{a}}$ injeção de insulina, respeitando-se a concentração de $40 \mathrm{mEq} /$ 1 no líquido de infusão. A quantidade necessária é de 02 a $04 \mathrm{mEq} / \mathrm{Kg}$ peso de KCL nas primeiras 24 $\mathrm{h}$, permitindo-se acréscimos que dependerão do nível sérico (até 5 a $6 \mathrm{mEq} / \mathrm{Kg}$ peso/24 h), com rigoroso controle laboratorial. Se o $\mathrm{K}+$ sérico for maior que $6 \mathrm{mEq} / \mathrm{l}$, suspender a infusão até a queda da potassemia.

d) Bicarbonato de sódio - Administrar $\mathrm{NaHCO}$, E.V. apenas se $\mathrm{pH}$ arterial for menor que 7,0 após início da reidratação e insulinoterapia, utilizandose uma dose de bicarbonato de sódio equivalente a $50 \%$ do déficit calculado (B.E. $\mathrm{x}$ peso corporal $\mathrm{x}$ 0,3 ) por via E.V., em 04 a $06 \mathrm{~h}$.

- Medir pH arterial 30 min após término da administração de bicarbonato. Se o pH continuar abaixo de 07,0 repetir a infusão.

\section{Cuidados Gerais}

- Sondagem nasogástrica com aspiração, se o paciente estiver com alteração significativa da consciência.

- Sondagem vesical, se o paciente estiver inconsciente ou não urinar após $6 \mathrm{~h}$ de tratamento.

- Oxigenação, se PO2 < $80 \mathrm{mmHg}$, respirando ar atmosférico. 
- Antibioticoterapia, assim que se evidenciem sinais de infecção.

- Se P.A. sistólica se mantiver abaixo de $80 \mathrm{mmHg}$ após $01 \mathrm{~h}$ de hidratação, avaliar a possibilidade de administração de outros expansores de volume.

\section{Controle}

a) Repetir glicosúria e cetonúria a cada $1 \mathrm{~h}$ até glicosúria + e cetonúria negativa, quando as dosa- gens poderão ser feitas a cada $02 \mathrm{~h}$.

b) Repetir glicemia, $\mathrm{Na}+\mathrm{e} \mathrm{K}+\mathrm{a}$ cada $02 \mathrm{~h}$.

c) PA e P de 30 em 30 min.

d) Temperatura a cada $02 \mathrm{~h}$.

e) Diurese horária.

Ë importante destacar que a agilidade no atendimento e a supervisão constante do médico são fundamentais para a boa evolução e garantia de melhor prognóstico, nos casos de CAD e EHH.

FREITAS-FOSS MC \& FOSS MC. Diabetic ketoacidosis and hyperosmolar hyperglycemic state. Medicina, Ribeirão Preto, 36: 389-393, apr./dec. 2003

ABSTRACT - Diabetic ketoacidosis is acute hyperglycaemic complication of DM type 1 patients and this situation of metabolic disturbances develops in a milieu of severe insulin deficiency, usually associated to stress conditions that increase the counterregulatory hormones. Hyperosmolar Hypergycemic state is a specific complication of the DM type 2 patients with predominance of the effects of hyperosmolarity and dehydration, specially involving the central nervous system. Precipitating factors, clinical presentation, basic pathophysiology and treatment of these diabetic hyperglycaemic emergencies are analysed in this review.

UNITERMS - Diabetes Mellitus Type 1. Diabetes Mellitus Type 2. Diabetic Ketoacidosis. Hyperosmolar Hyperglycemic State. Diabetes Mellitus, Insulin-Dependent. Diabetes Mellitus, NonInsulin-Dependent.

\section{BIBLIOGRAFIA RECOMENDADA}

1 - ALBERTI KGMM \& MARSHALL SM. Hyperglycaemic emergencies: A further look. In: ALBERTI KGMM \& KRALL LP, ed. The diabetes annual/6. Elsevier, New York p. 390-408, 1992.

2 - WALKER M; MARSHALL SM \& ALBERTI KGMM. Clinical aspects of diabetic ketoacidosis. Diabetes Metab Rev 5: 651663,1989

3 - ISRAEL RS. Diabetic ketoacidosis. Emerg Med Clin North Am 7: 859-871, 1989 .

4 - VIGNATI L; ASMAL AC; BLACK WL; BRINK SJ \& HARE JW. Coma in diabetes. In: MARBLE A; KRALL LP; BRADLEY RF; CHRISTLIEB AR \& SOELDNER JS, eds. Joslin's diabetes mellitus. $12^{\text {th }}$. ed. Lea-Eebriger, Philadelphia, p. 526552,1985
5 - FOSTER DN \& McGARRY JD. Acute complications of diabetes: Ketoacidosis, hyperosmolar coma, lactic acidosis In: DEGROOT LJ, ed. Endocrinology. W.B.Saunders, Philadelphia, v. 2, p. 1439-1453, 1989

6 - LAFFEL L. Ketone bodies: A review of physiology, pathophysiology and application of monitoring to diabetes. Diabetes Metab Res Rev 15: 412-526, 1999.

7 - KITABACHI AE; UMPIERREZ GE; MURPHY MB; BARRETT EJ; KREISBERG RA; MALONE JI \& WALL BM. Manegement of hyperglycemic crises in patients with diabetes. Diabetes Care 24:131-153, 2001.

8 - AMERICAN DIABETES ASSOCIATION. Hypreglycemic crises in patients with diabetes mellitus. Diabetes Care 24: 19881996, 2001. 